\title{
Guanylate-binding protein 1 participates in cellular antiviral response to dengue virus
}

\author{
Wen Pan ${ }^{\dagger}$, Xiangyang Zuo ${ }^{\dagger}$, Tingting Feng, Xiaohong Shi and Jianfeng Dai
}

\begin{abstract}
Background: Dengue virus (DENV), the causative agent of human Dengue hemorrhagic fever, is a mosquito-borne virus found in tropical and sub-tropical regions around the world. Vaccines against DENV are currently unavailable. Guanylate-binding protein 1 (GBP1) is one of the Interferon (IFN) stimulated genes (ISGs) and has been shown important for host immune defense against various pathogens. However, the role of GBP1 during DENV infection remains unclarified. In this study, we evaluated the relevance of GBP1 to DENV infection in in vitro model.
\end{abstract}

Findings: Quantitative RT-PCR (qRT-PCR) and Western blot showed that the expression of mouse Gbp1 was dramatically upregulated in DENV-infected RAW264.7 cells. The intracellular DENV loads were significantly higher in Gbp1 silenced cells compared with controls. The expression levels of selective anti-viral cytokines were decreased in Gbp1 siRNA treated cells, while the transcription factor activity of NF-kB was impaired upon GBP1 silencing during infection.

Conclusions: Our data suggested that GBP1 plays an antiviral role during DENV infection.

Keywords: GBP1, DENV, Antiviral response, NF-KB

\section{Findings}

Dengue virus (DENV), a member of the mosquito-borne flavivirus family, is an icosahedral, enveloped virus with a single-stranded positive sense RNA genome. Millions of cases of DENV infection occur worldwide each year $[1,2]$. Dengue hemorrhagic fever, the severe form of DENV infections, can cause serious haemorrhage, sudden drop in blood pressure (shock) and even death. Since no vaccine against DENV is currently available, much effort is needed to explore the host antiviral mechanisms for control of DENV infection and vaccine development [1,3-5].

Interferons (IFNs) are major antiviral cytokines released by host cells in response to viral and other pathogenic infections and play crucial roles in induction and regulation of both innate and adaptive immune responses [6]. IFNs establish an antiviral state by activating mainly the Janus kinase/signal transducer and activator of transcription (JAK/STAT) signaling pathway and

\footnotetext{
* Correspondence: daijianfeng@suda.edu.cn

${ }^{\dagger}$ Equal contributors

Institute of Biology and Medical Sciences, Jiangsu Key Laboratory of Infection and Immunity, Soochow University, Building 703, 199 Ren-ai Road, Suzhou 215123, P.R. China
}

(c) 2012 Pan et al.; licensee BioMed Central Ltd. This is an Open Access article distributed under the terms of the Creative Commons Attribution License (http://creativecommons.org/licenses/by/2.0), which permits unrestricted use, distribution, and reproduction in any medium, provided the original work is properly cited. are characterized as interferon-stimulated genes (ISGs), the roles of many ISGs during infection remain largely unknown $[7,8]$.

Guanylate-binding protein 1 (GBP1) is one of the ISGs that most strongly induced by IFNs [9] and belongs to a family of GTPases which are divided into three groups: (1) the large GTPases, also known as GBPs; (2) the small GTPases; (3) the Mx proteins. The human large GTPase family is composed of seven members encoded by a gene cluster located on chromosome $1[10,11]$. The emerging roles of GBP1 in host immune responses have been characterized in in vitro and in vivo models [12-15]. For example, GBP1 is overexpressed in endothelial cells upon activation of inflammatory cytokines and is involved in intestinal mucosal inflammation [16]. Recently, a study using Gbp1 knockout mice indicated that GBP1 has strong anti-bacterial activity [17]. There are also reports suggesting that GBP1 is involved in host immune response against chlamydia [18] and viruses including vesicular stomatitis virus, encephalomyocarditis virus and Hepatitis C Virus [19-21]. However, the distinct role of GBP1 during infection of other pathogens, including mosquito-borne flaviviruses remains largely 
unknown. We hereby investigated the physiological role of GBP1 during DENV infection in different in vitro models.

\section{Gbp1 is upregulated upon DENV infection}

Mouse macrophage cell line RAW264.7 can be readily infected by DENV, and served as an in vitro model for the study of host innate immune response to DENV. By using a quantitative RT-PCR (qRT-PCR) based small cDNA array (SABiosciences, Frederick, MD), we measured the expression profile of genes from Jak-Stat signaling pathway in DENV (DENV 2, New Guinea C strain) infected RAW264.7 cells. A number of genes were found up- or down-regulated upon DENV infection, as reported in our previous work (Additional file 1 of Ref. [22]). Among them, Gbp1 was upregulated 3.97fold in DENV infected cells in comparison to uninfected controls. Independent qRT-PCR and Western blotting further confirmed the upregulation of GBP1 (mRNA and protein) upon DENV infection (Figure 1, A and B). Recent studies have identified host genes that upregulated in human patients with acute DENV infection $[23,24]$. GBP1 was one of the genes most highly induced in patients' blood cells during early DENV infection [23], and this is consistent with our result here shown in in vitro study.

\section{Gbp1 has antiviral activity against DENV infection}

To study the specific role of GBP1 during DENV infection, an siRNA based RNA interference study was performed in RAW264.7 cells. siRNAs specific for Gbp1 or scrambled control (N.C.) were delivered into RAW264.7 cells respectively via electroporation. 24hrs after siRNA transfection, cells were challenged by DENV (MOI=1.0) for another 24hrs. Cells were then harvested and total RNA and cDNA were made according to standard protocols. Gbp1 was silenced efficiently as analyzed by qRT-PCR (Figure 2A) using gene specific primers (Table 1) and by Western blot (Figure 2B). The intracellular viral loads, in terms of the transcript levels of the envelop gene (E), were quantified by qRT-PCR and normalized to mouse beta-actin gene. As shown in Figure 2C, the DENV viral load was increased 5.0-fold $(p<0.05)$ in Gbp1 silenced cells compared with control cells. To measure the production of infectious virus from these cells, a plaque assay was performed in 293T cells. The titers of virus in supernatants from Gbp1 silenced cells were about 10- fold higher compared with that from control cells (Figure 2D). These data suggested that Gbp1 has an antiviral activity against DENV in RAW264.7 cells.

\section{Selective cytokines are downregulated in Gbp1 silenced cells upon DENV infection}

In order to examine whether the silencing of Gbp1 would affect the expression of cytokines, the expressions of some representative cytokines and chemokines were measured in Gbp1 siRNA or scrambled siRNA treated cells after DENV infection. Total of six genes were chosen for the study, including type I interferon (Ifn $\beta 1$ ), Interleukin-6 (Il6), Chemokine (C-X-C motif) ligand 1 (Cxcl1), Chemokine (C-X-C motif) ligand 2(Cxcl2), Chemokine (C-C motif) ligand $5(\mathrm{Ccl} 5)$ and $\mathrm{C}-\mathrm{C}$ chemokine receptor type $5(\mathrm{Ccr} 5)$. Gene specific primers and probe sets were purchased from Applied Biosystems (Carlsbad, CA). After DENV infection, the transcription levels of Ifn $\beta 1, I l 6$, and $C c l 5$ in Gbp1 silenced groups were decreased 2 to 3 -fold comparing with controls $(p<0.05)$. However, transcription levels of $C x c l 1 / 2$ and $C c r 5$ were not significantly affected (Figure 3, A-F). Consistent with the mRNA expression, the protein secretion of IFN $\beta$ and IL6 were decreased in Gbp1 silenced cells (Figure 3, G and $\mathrm{H})$. These data suggested that antiviral role of $G b p 1$

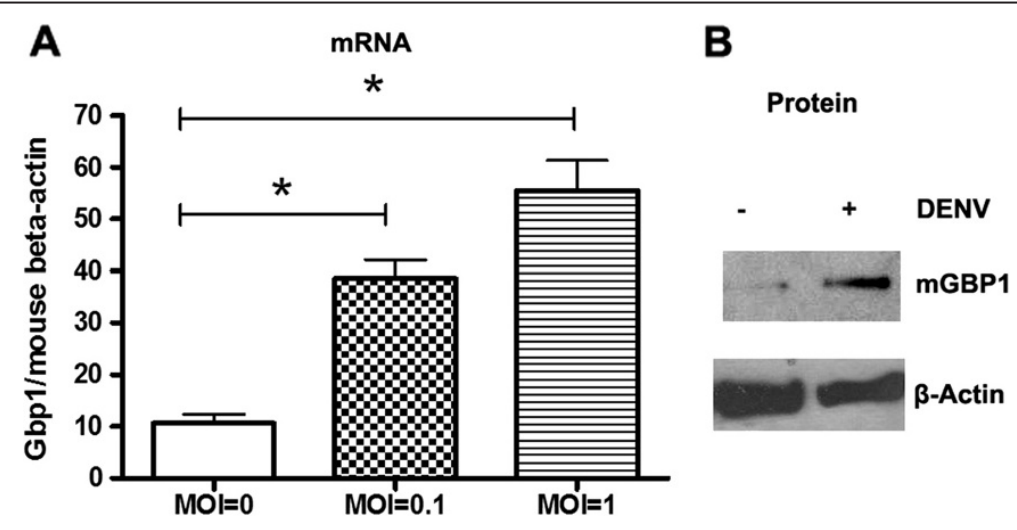

Figure 1 Gbp 1 is upregulated upon DENV infection. A. The gene expression was analyzed by quantitative RT-PCR (qRT-PCR), and normalized to mouse beta actin gene. Results are expressed as the mean + the SEM. ${ }^{*} p<0.05$ ( $t$-test). B. The protein level of mouse GBP1 was increased in DENV infected cells compared with uninfected cells, as shown in Western blot using anti- GBP1 antibody (sc 28579, Santa Cruz, CA). Representative results from at least 3 independent experiments. 


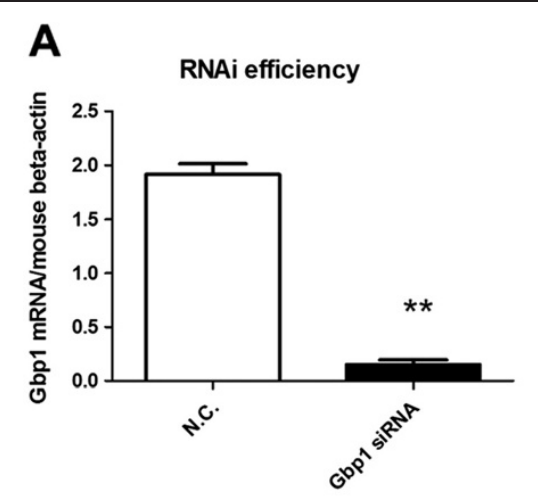

B
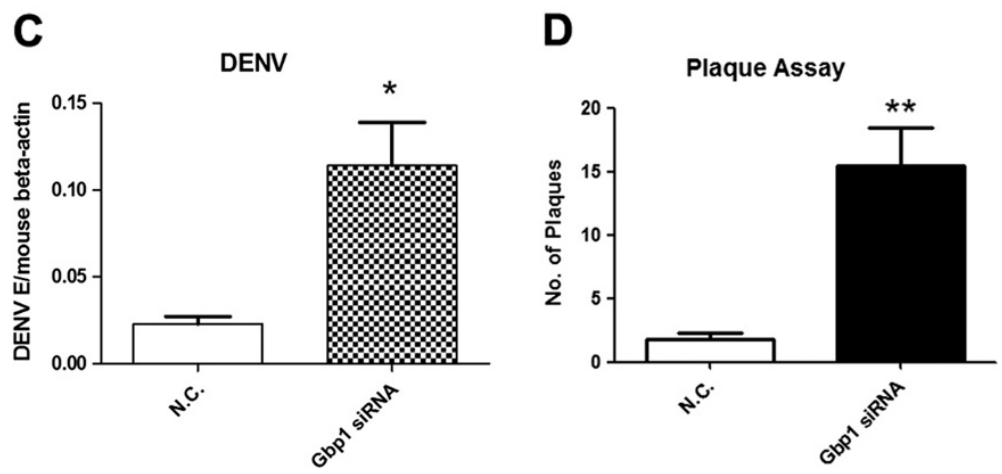

Figure 2 The viral burden is increased in Gbp1 silenced cells. A-B) RNAi efficiency for Gbp1 as shown in qRT-PCR (A) and Western blot (B). C-D) DENV burdens in RAW264.7 cells after RNAi silencing. C) The viral burdens were analyzed by measuring the virus E gene copy using qRT-PCR, and normalized to mouse beta actin gene. D) The titer of infectious DENV in supernatants of cells. Y axis represents the number of plaques formed in 293T cells when infected with viruses in $100 \mu \mathrm{l}$ of cell supernatants $24 \mathrm{~h}$ post infection. Results are expressed as the mean + the SEM. ${ }^{*} p<0.05$ and ${ }^{* *} p<0.01$ (t-test). Representative results from at least 3 independent experiments.

is associated with its ability to modulate some antiviral or pro-inflammatory cytokines during DENV infection.

\section{Transcriptional factor activity of NF-KB is impaired in} GBP1 silenced cells

NF- $\underline{\text { B }}$ (nuclear factor- kappa B) and AP1 (activator protein 1) play crucial roles in antiviral innate immune response through promoting the transcription of numerous antiviral or pro-inflammatory genes $[25,26]$. Since the expression levels of some cytokines were decreased in Gbp1 silenced cells after DENV infection, we further measured the activities of NF-kB and AP1 upon GBP1 silencing. 293 cells can be robustly infected by DENV [27], and are the most common system to measure the

Table 1 siRNA and oligo-primer sequences for this study

\begin{tabular}{|c|c|c|}
\hline No. & Sequence $\left(5^{\prime}-3^{\prime}\right)$ & Note \\
\hline 1 & GGAACGUAUAAAAGCAGAAtt & siRNA seq for mouse gene Gbp 1 \\
\hline 2 & AAGGCAUGUACCAUAAGCUtt & siRNA seq for human gene GBP1 \\
\hline 3 & AGAGGGAAATCGTGCGTGAC & Forward primer for qRT-PCR of mouse beta-actin \\
\hline 4 & CAATAGTGATGACCTGGCCGT & Reverse primer for qRT-PCR of mouse beta-actin \\
\hline 5 & GGGCATGGAGTCCTGTGGCA & Forward primer for qRT-PCR of human beta-actin \\
\hline 6 & GGGTGCCAGGGCAGTGATCTC & Reverse primer for qRT-PCR of human beta-actin \\
\hline 7 & CATTCCAAGTGAGAATCTCTITGTCA & Forward primer for qRT-PCR of DENV E gene \\
\hline 8 & CAGATCTCTGATGAATAACCAACG & Reverse primer for qRT-PCR of DENV E gene \\
\hline 9 & GGGCAGCTGTCTITGGGTAGAC & Forward primer for qRT-PCR of mouse Gbpl gene \\
\hline 10 & AGCATGAGGCCCTAGGAGCTGT & Reverse primer for qRT-PCR of mouse Gbpl gene \\
\hline 11 & AAGAGAGGACCCTCGCTCTTA & Forward primer for qRT-PCR of human GBP1 gene \\
\hline 12 & ATGCCTTGGTTAGGGGTGAC & Reverse primer for qRT-PCR of human GBP1 gene \\
\hline
\end{tabular}



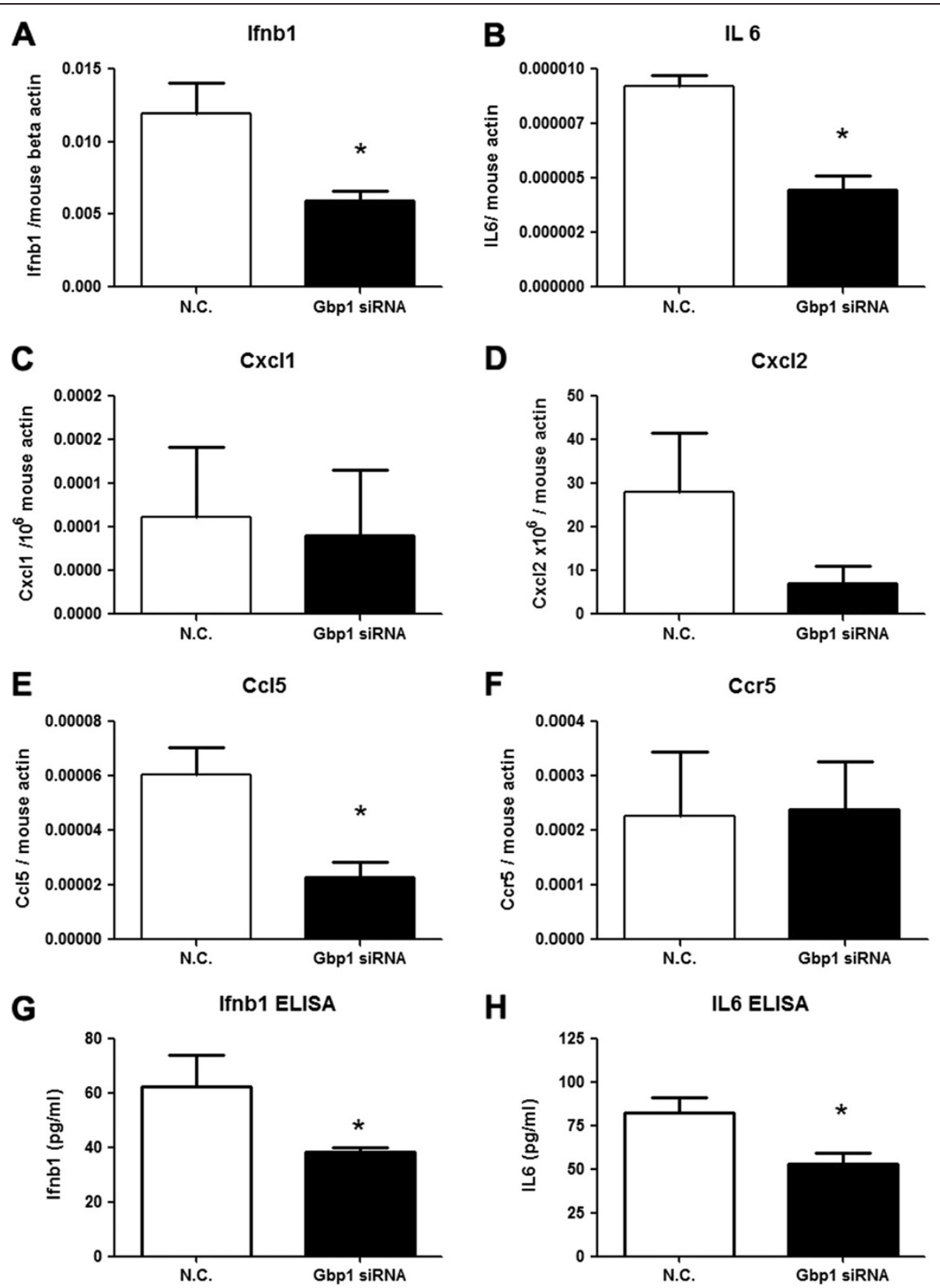

Figure 3 Cytokine expression in DENV infected cells after gene silencing. A-F) mRNA levels of selective cytokines/chemokines (A:Ifn $\beta$ 1, B:I/ 6, $\mathrm{C}:(x \mathrm{Cl} 1, \mathrm{D}: \mathrm{C} \times \mathrm{CI}$, E:CCI5,F:CCr5) were measured by qRT-PCR and normalized to mouse beta-actin gene. G-H) Protein level of IFN $\beta$ and IL6 in cell supernatants. Results are expressed as the mean + the SEM. ${ }^{*} p<0.05$ ( $t$-test). Representative results from at least 3 independent experiments.

transcriptional factor activity using dual luciferase reporter assay. Reporter plasmids pGL- NF-kB, pGL-AP1 and pRL-TK (internal control) (Promega, Madison, MI) in together with siRNAs specific for human GBP1 or scrambled control (N.C.), were delivered into 293T cells respectively by transfection using Lipofectamine ${ }^{\circledR}$ LTX \& PLUS (Invitrogen, Grand Island, NY). 24hrs after transfection, cells were challenged by DENV (MOI=1.0) for another $24 \mathrm{hrs}$, respectively. RNAi efficiency was confirmed by Western blot (Figure 4A). Cells were then harvested and lysed for dual luciferase reporter assay (Promega, Madison, MI). The relative activity of NF- $\mathrm{kB}$ was decreased by $40 \%$ in GBP1 knockdown cells in comparison with controls; while the AP1 activity was not significantly impaired in this case (Figure 4, B and C). These data suggested that GBP1 may influence activity of NF- $\mathrm{KB}$, thereby contribute to the production of antiviral or pro-inflammatory cytokines/chemokines. This could also partially address the mechanisms of how GBP1 inhibits DENV replication in cell culture. 


\section{A}
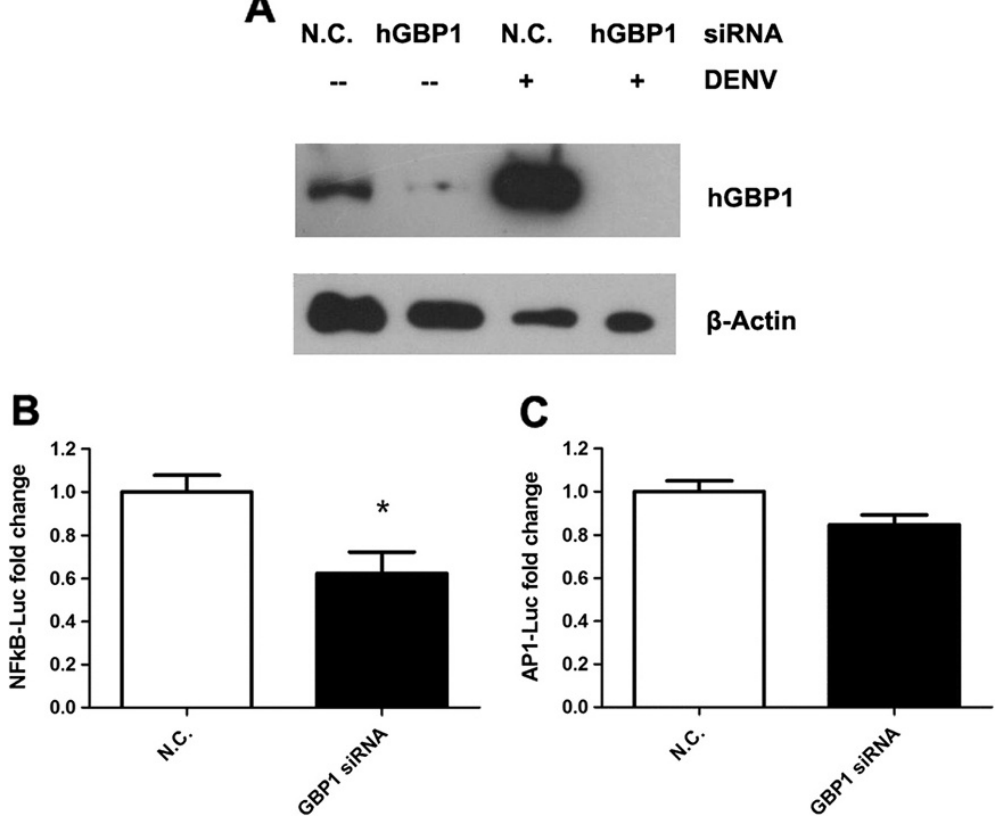

Figure 4 NF-KB activity is impaired upon GBP1 silencing. (A) GBP1 was silenced efficiently in 293T cells as shown in western blot. NF-KB (B) and AP1 (C) activities were measured by dual luciferase reporter assay. (The reporter activities were normalized by internal control (pRL-TK Renilla luciferase value). The mean value of activities from control cells were set to 1.0) Results are expressed as the mean + the SEM. ${ }^{*} p<0.05$ ( $t$-test). Representative results from at least 3 independent experiments.

More and more attention has been focused on the roles of large GTPase family in immune response [12-14]. GBP1 can be induced by IFN $\gamma$ as well as IFN $\alpha /$ $\beta$, and its induction can be augmented by TNF- $\alpha$, IL- 1 or LPS[12]. The inhibitory roles of GBP1 to different pathogens have been reported [17-19,21]. Itsui and his colleague showed that $\mathrm{HCV}$ replication was suppressed significantly by overexpression of GBP1, while binding of the HCV-NS5B protein to GBP1 countered the antiviral effect through inhibition of GTPase activity [21]. MacMicking speculated that GBP1 might help to limit the cell-to-cell spread of progeny virus through its anticell proliferative activity $[13,28]$. GBP1 has modest antiviral activity against the negative strand RNA viruses (such as Rhabdovirus, vesicular stomatitis virus), as well as the positive strand RNA viruses (Picornovirus and encephalomyocarditis virus) in cultured cells [19]. In both anti- chlamydia and antiviral study, the GTPase domain of GBP1 was suggested to be critical for its inhibitory activity $[18,21]$. A recent in vivo study further suggested that GBPs can solicit host defense proteins, including the phagocyte oxidase, antimicrobial peptides, and autophagy effectors, to kill intracellular bacteria [17]. While, more work are still needed to address the role and mechanisms of GBP1 in different infection models especially for viruses.

In this study, we confirmed that GBP1 shows inhibitory effect on DENV infection, influences the activity of
NF- $\mathrm{kB}$ and further contributes to the production of antiviral and pro-inflammatory cytokine/chemokines. This could be a novel mechanism for GBP1 to exert its antiviral activity in cellular level. Since NF- $\mathrm{BB}$ can be activated upon a broad range of pathogen infection, this could be a common action for GBP1 to play its role in different infection models. Further experiments will address the detail mechanisms of GBP1 on influencing $\mathrm{NF}-\mathrm{kB}$ pathway in various models.

\section{Abbreviations}

GBP1: Guanylate-binding protein-1; DENV: Dengue virus; Ifnß1: Type I interferon; II6: Interleukin-6; Cxcl1: Chemokine (C-X-C motif) ligand 1; Cxcl2: Chemokine (C-X-C motif) ligand 2; CCI5: Chemokine (C-C motif) ligand 5; Ccr5: C-C chemokine receptor type 5; NF-KB: Nuclear factor kappa B; AP1: Activator protein 1.

\section{Competing interests}

The authors declare that they have no competing interests.

\section{Authors contribution}

$J D, W P$ and XS designed the experiments and prepared the manuscript. $X Z$, WP and TF performed all the experiments. All authors read and approved the final manuscript.

\section{Acknowledgements}

This work was supported by a Project Funded by the Priority Academic Program Development of Jiangsu Higher Education Institutions (PAPD), Program for Changjiang Scholars and Innovative Research Team in University (PCSIRT) (IRT1075), National Natural Science Foundation of China (NSFC) $(81172812,81271792,31200648)$ and Jiangsu Natural Science Foundation (BK2012180). We thank Prof. Zhenyou Jiang from Jinan University,

Guangzhou, China for technical support. 
Received: 14 May 2012 Accepted: 15 November 2012

Published: 27 November 2012

\section{References}

1. Whitehorn J, Simmons CP: The pathogenesis of dengue. Vaccine 2011, 29:7221-7228

2. Gubler DJ: Epidemic dengue/dengue hemorrhagic fever as a public health, social and economic problem in the 21 st century. Trends Microbiol 2002, 10:100-103.

3. Murphy BR, Whitehead SS: Immune response to dengue virus and prospects for a vaccine. Annu Rev Immunol 2011, 29:587-619.

4. Fernandez-Garcia MD, Mazzon M, Jacobs M, Amara A: Pathogenesis of flavivirus infections: using and abusing the host cell. Cell Host Microbe 2009, 5:318-328

5. Durbin AP, Schmidt A, Elwood D, Wanionek KA, Lovchik J, Thumar B, Murphy BR, Whitehead SS: Heterotypic dengue infection with live attenuated monotypic dengue virus vaccines: implications for vaccination of populations in areas where dengue is endemic. $J$ Infect Dis 2011, 203:327-334

6. Malmgaard L: Induction and regulation of IFNs during viral infections. J Interferon Cytokine Res 2004, 24:439-454.

7. Rawlings JS, Rosler KM, Harrison DA: The JAK/STAT signaling pathway. Cell Sci 2004, 117:1281-1283.

8. Platanias LC: Mechanisms of type-I- and type-II-interferon-mediated signalling. Nat Rev Immunol 2005, 5:375-386.

9. Cheng YS, Colonno RJ, Yin FH: Interferon induction of fibroblast proteins with guanylate binding activity. J Biol Chem 1983, 258:7746-7750.

10. Prakash B, Praefcke GJ, Renault L, Wittinghofer A, Herrmann C: Structure of human guanylate-binding protein 1 representing a unique class of GTP-binding proteins. Nature 2000, 403:567-571.

11. Olszewski MA, Gray J, Vestal DJ: In silico genomic analysis of the human and murine guanylate-binding protein (GBP) gene clusters. J Interferon Cytokine Res 2006, 26:328-352.

12. Shenoy AR, Kim BH, Choi HP, Matsuzawa T, Tiwari S, MacMicking JD: Emerging themes in IFN-gamma-induced macrophage immunity by the p47 and p65 GTPase families. Immunobiology 2007, 212:771-784.

13. MacMicking JD: IFN-inducible GTPases and immunity to intracellular pathogens. Trends Immunol 2004, 25:601-609.

14. Naschberger $E$, Bauer $M$, Sturzl M: Human guanylate binding protein-1 (hGBP-1) characterizes and establishes a non-angiogenic endothelial cell activation phenotype in inflammatory diseases. Adv Enzyme Regul 2005, 45:215-227.

15. Lubeseder-Martellato C, Guenzi E, Jorg A, Topolt K, Naschberger E, Kremmer E, Zietz C, Tschachler E, Hutzler P, Schwemmle M, et al: Guanylate-binding protein-1 expression is selectively induced by inflammatory cytokines and is an activation marker of endothelial cells during inflammatory diseases. Am J Pathol 2002, 161:1749-1759.

16. Schnoor M, Betanzos A, Weber DA, Parkos CA: Guanylate-binding protein-1 is expressed at tight junctions of intestinal epithelial cells in response to interferon-gamma and regulates barrier function through effects on apoptosis. Mucosal Immunol 2009, 2:33-42.

17. Kim BH, Shenoy AR, Kumar P, Das R, Tiwari S, MacMicking JD: A family of IFN-gamma-inducible 65-kD GTPases protects against bacterial infection. Science 2011, 332:717-721.

18. Tietzel I, El-Haibi C, Carabeo RA: Human guanylate binding proteins potentiate the anti-chlamydia effects of interferon-gamma. PLoS One 2009, 4:e6499.

19. Anderson SL, Carton JM, Lou J, Xing L, Rubin BY: Interferon-induced guanylate binding protein-1 (GBP-1) mediates an antiviral effect against vesicular stomatitis virus and encephalomyocarditis virus. Virology 1999, 256:8-14.

20. Itsui Y, Sakamoto N, Kurosaki M, Kanazawa N, Tanabe Y, Koyama T, Takeda Y, Nakagawa M, Kakinuma S, Sekine $Y$, et al: Expressional screening of interferon-stimulated genes for antiviral activity against hepatitis $C$ virus replication. J Viral Hepat 2006, 13:690-700.

21. Itsui $Y$, Sakamoto $N$, Kakinuma S, Nakagawa M, Sekine-Osajima Y, TasakaFujita M, Nishimura-Sakurai Y, Suda G, Karakama Y, Mishima K, et al: Antiviral effects of the interferon-induced protein guanylate binding protein 1 and its interaction with the hepatitis C virus NS5B protein. Hepatology 2009, 50:1727-1737.
22. Dai J, Pan W, Wang P: ISG15 facilitates cellular antiviral response to dengue and west nile virus infection in vitro. Virol J 2011, 8:468.

23. Tolfvenstam T, Lindblom A, Schreiber MJ, Ling L, Chow A, Ooi EE, Hibberd $\mathrm{ML}$ : Characterization of early host responses in adults with dengue disease. BMC Infect Dis 2011, 11:209.

24. Hoang LT, Lynn DJ, Henn M, Birren BW, Lennon NJ, Le PT, Duong KT, Nguyen TT, Mai LN, Farrar JJ, et al: The early whole-blood transcriptional signature of dengue virus and features associated with progression to dengue shock syndrome in Vietnamese children and young adults. J Virol 2010, 84:12982-12994.

25. Pascual G, Glass CK: Nuclear receptors versus inflammation: mechanisms of transrepression. Trends Endocrinol Metab 2006, 17:321-327.

26. Ivashkiv LB: Inflammatory signaling in macrophages: transitions from acute to tolerant and alternative activation states. Eur J Immunol 2011, 41:2477-2481.

27. Diamond MS, Edgil D, Roberts TG, Lu B, Harris E: Infection of human cells by dengue virus is modulated by different cell types and viral strains. J Virol 2000, 74:7814-7823.

28. Guenzi E, Topolt K, Cornali E, Lubeseder-Martellato C, Jorg A, Matzen K, Zietz C, Kremmer E, Nappi F, Schwemmle M, et al: The helical domain of GBP-1 mediates the inhibition of endothelial cell proliferation by inflammatory cytokines. EMBO J 2001, 20:5568-5577.

\section{doi:10.1186/1743-422X-9-292}

Cite this article as: Pan et al:: Guanylate-binding protein 1 participates in cellular antiviral response to dengue virus. Virology Journal 2012 9:292.

\section{Submit your next manuscript to BioMed Central and take full advantage of:}

- Convenient online submission

- Thorough peer review

- No space constraints or color figure charges

- Immediate publication on acceptance

- Inclusion in PubMed, CAS, Scopus and Google Scholar

- Research which is freely available for redistribution 